\section{Case Report}

Joumal of Epilepsy Research pISSN 2233-6249 / eISSN 2233-6257

Received August 17, 2021

Revised September 1, 2021

Accepted December 6, 2021

Corresponding author:

Airenakho Emorinken, MBBS, FMCP

Department of Internal Medicine, Irrua

Specialist Teaching Hospital, KM 87 Benin

Auchi road, Irrua 310112, Nigeria

Tel. +234-905-476-9918

E-mail; emosairen@gmail.com

\title{
Metoclopramide-induced Acute Dystonia Misdiagnosed as an Epileptic Seizure in a Lupus Patient
}

\author{
Airenakho Emorinken, MBBS, FMCP' ${ }^{1}$ Oluwaseun Remi Agbadaola, MBBS, MWACP ${ }^{2}$ \\ ${ }^{1}$ Department of Internal Medicine, Irrua Specialist Teaching Hospital, Irrua, Edo State, Nigeria; ${ }^{2}$ Department of \\ Internal Medicine, Al jawal General Hospital, Uyun Al-jawal, Saudi Arabia
}

\begin{abstract}
Acute dystonic reactions are the most prevalent extrapyramidal adverse effects associated with metoclopramide. It could be mistaken for a variety of other conditions, such as seizures, tetanus, and encephalitis, to name a few possibilities. We present a case of a 26 -year-old female misdiagnosed as having an epileptic seizure who was rushed to the emergency unit with an involuntary bilateral upward deviation of the eyes, spasm, stiffness, lateral deviation of the neck, and protrusion of the tongue. Symptoms occurred 36 hours after the commencement of metoclopramide, used to treat nausea and vomiting in the referring hospital. All the laboratory work was normal. The drug was discontinued and $5 \mathrm{mg}$ of intravenous biperiden was administered. The symptoms subsided in about 10 minutes with no recurrence. Metoclopramide-induced acute dystonia not only creates an anxious environment for patients but may also be life-threatening. Due to the high probability of misdiagnosis, detailed drug history and a high index of suspicion are critical in making the correct diagnosis. (2021;11:156-158)
\end{abstract}

Key words: Metoclopramide, Dystonia, Biperiden, Seizure

\section{Introduction}

Dystonia is a movement disorder marked by involuntary, continuous, or intermittent muscle contractions of various groups of muscles, leading to aberrant, frequently repetitive movements and/or postures. ${ }^{1}$ It is usually patterned, twisted, and tremulous. ${ }^{1}$ Acute dystonia is the most prevalent form of extrapyramidal side effect associated with metoclopramide use. ${ }^{2}$ Symptoms can appear hours or days after metoclopramide use and can be misdiagnosed as tetanus, hypocalcemia, epileptic seizures, or even meningoencephalitis. ${ }^{3}$ The standard of care is to discontinue the offending drug and administer an anticholinergic or antihistaminic drug parenterally. ${ }^{3,4}$ We present a case of metoclopramide-induced acute dystonic reaction misdiagnosed as an epileptic seizure in a lupus patient.

\section{Case Report}

A 26-year-old female was referred to the emergency unit as a case of an epileptic seizure. She presented to the referring facility with persistent nausea and vomiting, for which she was commenced on intravenous metoclopramide $10 \mathrm{mg}$ every 8 hours. Thirty-six hours after initiating metoclopramide and receiving a cumulative dose of $40 \mathrm{mg}$, she began experiencing paroxysms of painful muscle spasms and stiffness in her neck muscles, as well as protrusion of her tongue. Additionally, she had double/blurred vision and involuntary upward and lateral deviation of the eyes. The paroxysms lasted between one and 2 minutes, with spontaneous resolution occurring at 3-to 5-minute intervals. She was completely aware of her circumstances and surroundings and remained conscious during and between paroxysms. There was no history of head trauma, fever, skin breaks or injuries, or tooth or ear infections. The patient was tetanus-immunized. Her social and family history were unremarkable. She was a known lupus nephritis patient who had been stable for 2 years on mycophenolate mofetil and hydroxychloroquine.

She was apprehensive and conscious on examination, with a Glasgow Coma Score of 15. Her neck hyperextended and deviated to the left, with her tongue protruding. Her pupils were equal and reactive to light bilaterally. Her eyes were deviated upward and laterally. She had a pulse rate of 88 beats per minute, blood pressure of $120 / 80 \mathrm{mmHg}$, a respiratory rate of 20 cycles per minute, oxygen saturation of $98 \%$ in ambient air, and a temperature of $37.2^{\circ} \mathrm{C}$. Other aspects of her physical examination were otherwise unremarkable. The results of laboratory tests such as a complete blood count, erythrocyte sedimentation rate, serum calcium, electro- 
lyte, urea, and creatinine were all normal. On the basis of these features and prior use of metoclopramide, a diagnosis of metoclopramide-induced acute dystonia was made. She was given $5 \mathrm{mg}$ of biperiden intravenously and metoclopramide was discontinued. After about 10 minutes, her dystonic symptoms completely resolved with no recurrence. She was advised to abstain from further use of metoclopramide and was discharged the next day. Her follow-up clinic visit a week later was uneventful.

\section{Discussion}

Metoclopramide is a selective dopamine $\left(D_{2}\right)$ receptor antagonist that is often used to treat gastrointestinal and neurological conditions, including nausea, vomiting, gastroesophageal reflux disease, gastroparesis, and neurogenic bladder. ${ }^{5,6}$ It is a frequently prescribed and affordable anti-emetic. ${ }^{5}$ It has a relatively short half-life and also acts as a $5-\mathrm{HT}_{3}$ antagonist and a $5-\mathrm{HT}_{4}$ agonist. ${ }^{5}$ Dopamine stimulates the medullary chemoreceptor trigger zone, which induces nausea and vomiting. Metoclopramide's anti-emetic properties are due to its ability to inhibit $\mathrm{D}_{2}$ and $5-\mathrm{HT}_{3}$ receptors in the chemoreceptor trigger zone. ${ }^{5}$ Metoclopramide's antagonistic effect on dopamine receptors in the basal ganglia disrupts the dopaminergic-cholinergic balance, resulting in decreased central dopamine transmission and increased acetylcholine release relative to dopamine. ${ }^{5,6}$ This effect on the basal ganglia is associated with extrapyramidal side effects, including acute dystonia, tardive dyskinesia, akathisia, and drug-induced parkinsonism. ${ }^{5,6}$

Acute dystonic reactions due to metoclopramide use have been reported to occur in $0.2-25.0 \%$ of patients. ${ }^{7.8}$ Little has been done regarding research on metoclopramide-induced dystonic reactions in the developing world, which is surprising given the medication's increased prescriptions and accessibility as an over-the-counter anti-emetic. Due to the dearth of data collection and under-reporting of cases, the incidence of these reactions is underestimated. In a study conducted in a teaching hospital in southwest Nigeria, Babatola et al. ${ }^{9}$ found that $0.48 \%$ of the juvenile population had drug-induced dystonia, with $11.8 \%$ of them caused by metoclopramide.

Symptoms of dyskinesia appear within the first 24-72 hours after using metoclopramide. ${ }^{10}$ These adverse events tend to occur more with higher drug doses in the pediatric population, females, and individuals younger than 30 years of age. ${ }^{11}$ It is still unclear why females are at a higher risk of developing dystonic reactions. ${ }^{12}$ According to several studies, metoclopramide-induced adverse effects are dose-in- dependent and unrelated to blood drug concentrations. ${ }^{13,14}$ However, the drug is also reported to have an accumulative effect when repeated doses are administered. ${ }^{15}$ According to van der Padt et al., ${ }^{16}$ metoclopramide-induced acute dystonic reactions can also occur in people with a CYP450 2D6 genetic polymorphism. Therefore, prescribing the drug with caution in individuals with a family history of acute dystonic reaction is advised. Our patient, a 26-year-old female, developed acute dystonia 36 hours after receiving her first dose of metoclopramide. Three of the four risk factors outlined above were present in her case.

Dystonia is typically manifested by involuntary movements of the limbs, facial grimacing, torticollis, oculogyric crisis, rhythmic protrusion of the tongue, opisthotonos, trismus, bulbar type of speech, and rarely by laryngospasm, which is life-threatening. ${ }^{17,18}$ Delayed manifestations, such as tardive dyskinesia, are more common with prolonged treatment. The index patient presented with an acute dystonic reaction manifested by torticollis, tongue protrusion, and an oculogyric crisis. Metoclopramide-induced acute dystonic reactions are treated by discontinuing metoclopramide and administering parenteral anticholinergic or antihistaminic medications, frequently biperiden, benztropine, and diphenhydramine. ${ }^{3}$ The preferred route is intravenous, with signs and symptoms disappearing within 10 minutes. In the event that an intravenous line cannot be obtained, the intramuscular route is an alternative. However, it takes 30 minutes to absorb. ${ }^{4}$ Due to substantial gut first-pass metabolism and limited absorption, the oral route is the most unreliable. ${ }^{19}$ Benzodiazepines like diazepam induce immediate muscle relaxation and can be used in combination with anticholinergics. ${ }^{16}$ Our patient was successfully treated with a single intravenous dosage of biperiden, and her symptoms resolved in about 10 minutes.

Recognizing drug-induced acute dystonia can be difficult, even more so in our setting, due to the high likelihood of misdiagnosis. ${ }^{20,21}$ Acute dystonic reactions are frequently misdiagnosed as tetanus, meningitis, convulsions, encephalitis, strychnine poisoning, hypocalcemia, electrolyte abnormalities, and conversion disorders. ${ }^{14,20}$ Our patient was referred with a misdiagnosis of an epileptic seizure. This can result in a waste of resources, an increase in patient hospital stay, and may influence decisions relating to diagnosis. As a result, doctors should be familiar with the clinical presentation of acute dystonia as well as other conditions that should be evaluated in the differential diagnosis. A detailed drug history, including the use of over-the-counter medications, is critical in establishing an accurate diagnosis. If there is any ambiguity about the diagnosis, it is reasonable to treat it as a medication-induced acute dystonic reaction and then investigate further if there is no re- 
sponse to treatment. The clinician's most important diagnostic tool continues to be the ability to extract information that will aid in establishing the correct diagnosis. ${ }^{20}$

Metoclopramide is a commonly used antiemetic with significant adverse effects, the most notable of which is acute dystonia, as demonstrated in this case. This not only creates an anxious environment for patients, but it may also be life-threatening. An accurate diagnosis requires eliciting a detailed drug history. Clinicians may have difficulty recognizing dystonia and thus must maintain a high level of clinical suspicion.

\section{Conflicts of Interest}

The authors declare that they have no conflicts of interest.

\section{References}

1. Albanese $A$, Bhatia $K$, Bressman SB, et al. Phenomenology and classification of dystonia: a consensus update. Mov Disord 2013;28:863-73.

2. Guala A, Mittino D, Ghini T, Quazza G. Are metoclopramide dystonias familial? Pediatr Med Chir 1992;14:617-8.

3. El Ç, Çelikkaya ME. Varied clinical presentations of acute dystonic reaction due to metoclopramide. Pediatr Emerg Care 2019;35:369-72.

4. Tianyi FL, Agbor VN, Njim T. Metoclopramide induced acute dystonic reaction: a case report. BMC Res Notes 2017;10:32.

5. Rao AS, Camilleri M. Review article: metoclopramide and tardive dyskinesia. Aliment Pharmacol Ther 2010;31:11-9.

6. Ganzini L, Casey DE, Hoffman WF, McCall AL. The prevalence of metodopramide-induced tardive dyskinesia and acute extrapyramidal movement disorders. Arch Intern Med 1993;153:1469-75.

7. Jo YY, Kim YB, Yang MR, Chang YJ. Extrapyramidal side effects after metoclopramide administration in a post-anesthesia care unit-a case report-. Korean J Anesthesiol 2012;63:274-6.

8. Valkova M, Stamenov B, Peychinska D, Veleva I, Dimitrova P, Radeva
P. Metoclopramide-induced extrapyramidal signs and symptoms-brief review of literature and case report. J IMAB 2014;20:539-41.

9. Babatola AO, Taiwo AB, Ogundare EO, et al. Pattern and outcome of drug-induced dystonia at the paediatric emergency room of a teaching hospital in southwestern Nigeria. J Med Sci 2020;20:13-7.

10. Bateman DN, Rawlins MD, Simpson JM. Extrapyramidal reactions with metoclopramide. Br Med J (Clin Res Ed) 1985;291:930-2.

11. Geyer HL, Bressman SB. The diagnosis of dystonia. Lancet Neuro/ 2006; 5:780-90.

12. Isikay S, Yilmaz $\mathrm{K}$, Almancioglu M. Evaluation of patients with metoclopramide-induced acute dystonic reaction. J Acad Emerg Med 2013;12:80-4.

13. Bateman DN, Craft AW, Nicholson E, Pearson AD. Dystonic reactions and the pharmacokinetics of metoclopramide in children. $\mathrm{Br} / \mathrm{Clin}$ Pharmacol 1983;15:557-9.

14. Arumugam J, Vijayalakshmi AM. Metoclopramide-induced oculogyric crisis presenting as encephalitis in a young girl. Indian J Pharmacol 2012;44: 266-7.

15. Akbuga-Ozel B, Aksel G, Kilicli E, et al. Metoclopramide-induced acute dystonic reaction misinterpreted as conversion disorder and seizure. Eur J Gen Med 2017;14:122-3.

16. van der Padt A, van Schaik RH, Sonneveld P. Acute dystonic reaction to metoclopramide in patients carrying homozygous cytochrome P450 2 D6 genetic polymorphisms. Neth J Med 2006;64:160-2.

17. Karagoz G, Kadanali A, Dede B, Anadol U, Yucel M, Bektasoglu MF. Metoclopramide-induced acute dystonic reaction: a case report. Eurasian J Med 2013;45:58-9.

18. Freudenreich 0. Atypical laryngeal dystonia caused by an antiemetic. Am Fam Physician 2004;69:1623.

19. Huang SM, Athanikar NK, Sridhar K, Huang YC, Chiou WL. Pharmacokinetics of chlorpheniramine after intravenous and oral administration in normal adults. Eur J Clin Pharmacol 1982;22:359-65.

20. Dingli $K$, Morgan $R$, Leen C. Acute dystonic reaction caused by metodopramide, versus tetanus. BMJ 2007;334:899-900.

21. Ferrando SJ, Eisendrath SJ. Adverse neuropsychiatric effects of dopamine antagonist medications. Misdiagnosis in the medical setting. Psychosomatics 1991;32:426-32. 